\title{
Feedback Regulation of Immune Response to Maximum Exercise in Gulf War IIIness
}

\author{
Cole A. Lyman ${ }^{\dagger}$ \\ Center for Clinical Systems \\ Biology \\ Rochester General Hospital \\ Rochester, NY, USA \\ Computer Science Department \\ Brigham Young University \\ Provo, UT, USA \\ cole@colelyman.com \\ Mary Fletcher \\ Dept. of Clinical Immunology \\ EM Paper Laboratory of Clinical \\ Immunology \\ Inst. for Neuroimmune Medicine \\ Nova Southeastern University \\ Fort Lauderdale, FL, USA
}

\author{
Mark Clement \\ Computer Science Department \\ Brigham Young University \\ Provo, UT, USA
}

Nancy G. Klimas

Dept. of Clinical Immunology

Inst. for Neuroimmune Medicine

Nova Southeastern University

Fort Lauderdale, FL, USA

Miami Veterans Affairs Medical Center

Miami, FL, USA

\author{
Travis J. A. Craddock \\ Dept. of Psychology and \\ Neuroscience, Computer Science, \\ and Clinical Immunology \\ Clinical Systems Biology Group, \\ Inst. for Neuroimmune Medicine \\ Nova Southeastern University \\ Fort Lauderdale, FL, USA
Gordon Broderick Center for Clinical Systems Biology
Rochester General Hospital
Rochester, NY, USA
Dept. of Biomedical Engineering,
Rochester Institute of Technology,
Rochester, NY, USA
Gordon.Broderick@rochesterregio nal.org

\begin{abstract}
Gulf War Illness is a disease that affects about a third of the veterans that served in the 1990-91 Persian Gulf War. The symptoms are varied, the cause is unknown, and there is no known treatment. In this paper we compare the dynamic characteristics of cytokines feedback response to exercise in $n=12$ veterans diagnosed with Gulf War Illness (GWI) and $n=12$ healthy veterans deployed to the same theatre of operations. A third-order transfer function is used to model the process dynamics of 18 cytokines projected onto principal components with a Proportional Integral Derivative (PID) controller enforcing the homeostatic regulation of these co-expression patterns. Transfer functions were fit to each subject for each principal component and the dynamic response parameters compared between the veteran control and GWI subject groups. Results of this analysis indicate that while much of the regulatory response dynamics are shared by both groups, there is a significant difference in the damping of the Th1:Th2 cytokine response to exercise in Gulf War Illness.
\end{abstract}

†Corresponding author

Permission to make digital or hard copies of part or all of this work for personal or classroom use is granted without fee provided that copies are not made or distributed for profit or commercial advantage and that copies bear this notice and the full citation on the first page. Copyrights for components of this work owned by others than ACM must be honored. Abstracting credit is permitted. To copy otherwise, of republish, to post on servers or to redistribute to lists, requires prior specific permission and/or a fee. Request permissions from Permissions@acm.org. ACM-BCB '19, September 7-10, 2019, Niagara Falls, NY, USA

(C) 2019 Association of Computing Machinery.

ACM ISBN 978-1-4503-6666-3/19/09...\$15.00

https://doi.org/10.1145/3307339.3342188

\section{KEYWORDS}

Exercise response; immune dynamics; feedback control; Gulf War Illness; oscillatory behavior

\section{ACM Reference format:}

Cole A. Lyman, Mark Clement, Travis J. A. Craddock, Mary Fletcher, Nancy G. Klimas and Gordon Broderick. 2019. Feedback Regulation of Immune Response to Maximum Exercise in Gulf War Illness In Proceedings of $10^{\text {th }}$ ACM International Conference on Bioinformatics, Computational Biology and Health Informatics (ACM-BCB '19), September 7-10, 2019, Niagara Falls, NY, USA. ACM, New York, NY, USA, 6 pages. https://doi.org/10.1145/3307339.3342188

\section{Introduction}

Gulf War Illness (GWI) is characterized as a set of nonspecific symptoms reported more frequently by veterans deployed in the Persian Gulf War (1990 to 1991) than by veterans of other conflicts. Veterans are diagnosed with GWI if they experience at least one chronic symptom from at least two of the following general categories: fatigue, mood-cognition, and musculoskeletal [1]. In 2010, approximately 250,000 of the almost 700,000 soldiers deployed during the Gulf War suffered from GWI [2]. Furthermore, a longitudinal study found that this number increased over the 10 years following their return from the war theatre [3], indicating that symptoms may have a late and gradual onset. The cause of GWI is unknown, but exposure to the organophosphate chemical warfare agents sarin and cyclosarin are suspected [4-5]. Many of the symptoms associated with GWI appear to involve altered regulation of the immune system [6]. 
Many alterations in immune system behavior can be observed through changes in the expression of cytokines, which are proteins that act as the chemical language of the immune system [7]. Abnormalities in cytokine trafficking have been associated with the persistence of GWI [8] and can be assessed by experimentally controlled induction of stress via a maximum exercise challenge [9-12]. In this paper we analyze the levels of 18 cytokines measured in $n=12$ GWI subjects and a control group of $\mathrm{n}=12$ healthy sedentary Gulf War era veterans obtained from peripheral blood plasma sampled at 9 time points before during and after maximal exercise challenge. In order to identify characteristic differences in how the immune system responds to physiological challenge, the parameters of a feedback control transfer function were fit to these experimental time course data for each individual and parameter values compared across subject groups. Results of this comparison indicate that while subjects in both groups share much of the same immune response dynamics, subjects in the GWI group typically display a more sluggish coregulation of inflammatory cytokines relative to their antiinflammatory counterparts.

\section{Methods}

\subsection{Experimental Data}

Subjects participating in this study were all male veterans, 40 to 59 years of age, that served in Operation Desert Storm. Control and illness groups were similar in demographic profile, fitness level and body mass index. Cytokine expression levels were measured in peripheral blood plasma from 24 males, 12 of which were diagnosed with GWI and 12 healthy control Gulf War ear veterans. We surveyed the expression of 18 different cytokines using an ELISA-based assay in peripheral blood plasma samples collected at 9 time points (Table 1) during a graded maximal exercise challenge. In this time course the third sample was collected at maximum exertion (maxVO2) whereupon the subject was removed from the stationary bicycle and allowed to recover.

\subsection{Data Preprocessing}

The cytokine levels are first $z$-transformed, that is centered and range adjusted to have mean, $\mu=0$, and variance, $\sigma=1$. Next, cytokine co-expression features are extracted from the data (both GWI subjects and controls) using Principal Component Analysis (PCA) [13] which captures cytokine interactions as weighted sums of their expression values or latent vectors that are mutually orthogonal. Importantly, these PCA features were extracted across all individuals and as such represent cytokine interaction patterns that are common to both groups. While these interaction patterns are illness agnostic in structure, we expect that they will be over or under represented in one phenotypic group versus the other and that their response dynamics will differ. Furthermore, transforming the data via PCA allows for the decomposition of response dynamics into progressively finer levels of resolution. As such the earlier features, e.g. PC 1, essentially filter out coarse similarities in the data allowing the groups to be separated on the basis of more subtle differences more readily.

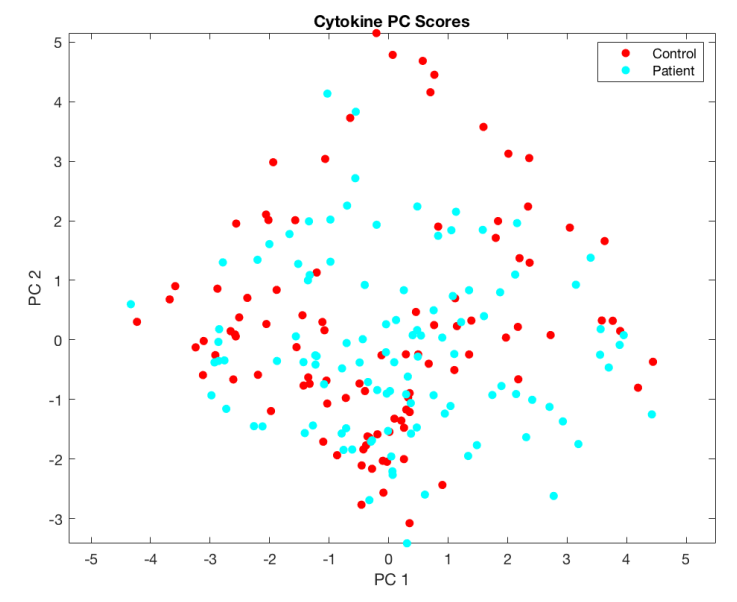

Figure 1: The normalized and PCA transformed cytokine levels for all time points and all individuals (except for the outlier). In the first two PCs, there is no visible separation between control and GWI subject samples.

In a first screening, one subject from the control group was removed from further analysis as this individual's cytokine levels were significantly different from that of all others. With this outlier removed, estimation of the PCA model produced the decomposition of overall variation (in both control and patient subjects) explained by each Principal Component (PC) show in Table 2. The first two PCs explained approximately $33 \%$ of the overall variability in the data. Samples from all time steps and all individuals (except for the outlier) are shown in Figure 1, plotted in the space of the first and second co-expression features (PCs). At this level of resolution there is no clear separation in the cytokine co-expression levels in samples from control and GWI subjects. This could imply that GWI subjects may differ not in terms of absolute co-expression, but rather in terms of the temporal response of these patterns to exercise challenge. To test this hypothesis, the score or representation of each co-expression feature (PC) at each time point was fit to a closed loop transfer function in order to extract the dynamic response parameters describing immune response to challenge in each GWI and veteran control subject.

To facilitate identification of the closed loop transfer function, the data were first interpolated with the piecewise cubic Hermite method [14] according to the measurement times to produce a uniformly sampled time course. These interpolated and resampled time courses were truncated at the T2 time point. This is done because the T3 time point is 24 hours away from the initial measurement and is intended to be used as a return to the initial resting state. However, this point is not included after interpolation because this analysis is focused on the short-term response to stress, but it is included in the interpolated values to improve the accuracy of the interpolation. Finally, all cytokine PC co-expression scores were shifted vertically by the amount of the initial measurement. This is done so that the output of the system is initially at 0 , and it does not change the inherent dynamics of the cytokine signal.

Lastly, an external input $u(t)$, was assembled to represent the magnitude of the exercise disturbance for each individual. 
Disturbances are set to 0 at all times except for a ramp starting at T0 up to a value of 1 at T1. Each of the inputs is constructed relative to the time at which the individual reached peak exercise (T1) (see Table 1)

Table 1: Target and approximate sample collection times (irregularly spaced) at and the corresponding exercise state. Exact times for each individual are used in the analysis.

\begin{tabular}{lll} 
Target Time & Approx. Elapsed Time & State \\
\hline T0 & 0 & Resting \\
T0+3 & $10 \mathrm{~min}$. & Intermediate exercise \\
T1 & $20 \mathrm{~min}$. & Peak exercise \\
T1+10 & $30 \mathrm{~min}$. & Resting \\
T1 +20 & $40 \mathrm{~min}$. & Resting \\
T1 +30 & $50 \mathrm{~min}$. & Resting \\
T1 +60 & $1 \mathrm{~h} .20 \mathrm{~min}$ & Resting \\
T2 & $4 \mathrm{~h} .20 \mathrm{~min}$ & Resting \\
T3 & $24 \mathrm{~h}$. & Resting
\end{tabular}

\subsection{Model Class Selection and Parameter Fitting}

In order to adequately select a model class, there are some considerations about the nature of the data that must be accounted for. When choosing an appropriate model class for this analysis it was useful to consider some of the properties of cytokines. For example, cytokines are pleiotropic, partially redundant, and synergistic; thus, resulting in complex behavior [7, 15-16]. Accordingly, the model class must be expected to adequately account for higher-order complex response behaviors.

Accordingly, in this analysis a fourth-order underdamped transfer function was chosen as the process feedback control model for the immune system (see Equation 1). This model class was chosen because it has a small number of parameters and yet can generalize to the more complex interactions that are associated with cytokines. Note that higher order transfer functions would require more parameters and therefore more training data to adequately fit the model.

$$
G(s)=\frac{K_{p}}{s\left(1+2 \zeta T_{w} s+\left(T_{w} s\right)^{2}\right)} * \frac{\left(1+T_{z} s\right)}{\left(1+T_{p 3} s\right)}
$$

There are 5 parameters in this transfer function that are fit to the PC cytokine scores: overall gain $K_{p}$, second-order process time constant $T_{w}$, damping factor $\zeta$, controller time constant $T_{p 3}$, and zero time constant $T_{z}$. Bounds are placed on the parameters to help with the highly non-linear parameter fitting over a complex landscape containing multiple local optima as well as to increase the biological relevance of the models. For example, $0.1 \leq \zeta \leq$ 0.9 constrains the models to be underdamped and $-5 \leq K_{p} \leq 5$ was determined by the minimum and maximum cytokine feature scores in all PCs. The bounds for each of the time constants $\left(T_{w}\right.$, $T_{p 3}$, and $T_{z}$ ) is $0 \leq T \leq 100$. The data were fit using the System Identification Toolbox in MATLAB, release 2019A (MathWorks, Natick, MA).

\subsection{Validation}

Model parameter values obtained for each of the healthy subjects are compared to the parameter values found for each of the GWI subjects using the non-parametric Mann-Whitney U test [17]. This rank-based test will indicate which distribution of parameters in each PC are statistically different between subject groups. Here, a null p-value $<0.05$ is considered statistically significant.

Goodness of model fit is measured using the normalized root mean square error (NRMSE) (Equation 2).

$$
f i t=1-\frac{\|x-\hat{x}\|}{\|x-\bar{x}\|}
$$

Where $x$ is a vector of training data, $\hat{x}$ is a vector of predicted values, and $\bar{x}=\frac{1}{n} \sum_{i=0}^{n} x_{i}$ is the average of the training data $x$ where $n$ is the number of training data points. The fit percentages for the models in each PC are shown in Figure 3.

Table 2: Percentage overall variability in the expression of 18 cytokines explained in each PC feature. Notice the increasingly shallow decrease in variation explained from PC 4 onward.

\begin{tabular}{ll} 
Principal Component & Percentage Explained \\
\hline 1 & 19.0881 \\
2 & 14.4606 \\
3 & 9.4789 \\
4 & 8.1154 \\
5 & 7.0032 \\
6 & 6.4987 \\
7 & 5.0663 \\
8 & 4.5124 \\
9 & 3.7964 \\
10 & 3.6109 \\
11 & 3.3219 \\
12 & 3.0106 \\
13 & 2.7451 \\
14 & 2.5750 \\
15 & 2.3282 \\
16 & 1.8533 \\
17 & 1.4976 \\
18 & 1.0373
\end{tabular}

\section{Results}

Null probabilities for the Mann-Whitney test comparing parameter values across groups are presented in Table 3. There are two parameters with p-values $<0.05$, namely the dampening coefficient $\zeta$ in PC 4 and the overall gain $K_{p}$ in PC 9. In other words, at the resolution of PC 4 , the coefficient $\zeta$ is significantly different in the control group than in the GWI subject group. Recall that the $\zeta$ controls how much the response will oscillate. Figure 4 illustrates the difference in the magnitude of oscillations between the median trajectories in PC 4 for GWI and control subjects. Notice the significant difference at this level of detail in the combined cytokine response trajectory (Figure 4) even though there was no significant difference in the magnitude of combined cytokine expression at coarser levels of resolution (PC 1 and 2 in 
Figure 1). Differences in the distributions of $\zeta$ values in PC 4 between the subject groups are shown in Figure 5.

The models capture the dynamics of the PC values which are a linear combination of the original cytokine levels. In order to draw informed conclusions about the underlying biological kinetics of a PC feature, one must analyze the corresponding feature weights for each cytokine. One sample was left out and the PCA was computed on the remaining samples; this was repeated for each of the samples. The mean cytokine weights for PC 4 are shown in Figure 6 with the standard error computed using a leave-one-out cross-validation where each sample is omitted in turn and the PC weights iteratively computed on the remaining samples. Additionally, a one-sample t-test is performed on the distribution of each cytokine weight to test if the mean is statistically equivalent to 0 , signifying that the cytokine has essentially no representation in the PC (red bars in Figure 6). The raw p-values were adjusted for multiple comparison using the BenjaminiHochberg method [18] and these adjusted p-values are used in Figure 6.

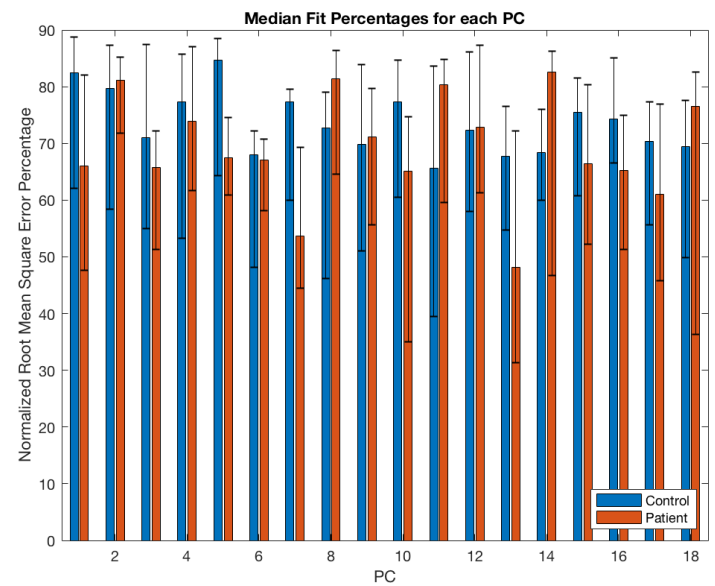

Figure 3: Median percentages fit of the models as calculated by the normalized root mean square error (NRMSE). The error bars represent the $25 \%$ and $75 \%$ interquartile range. The relatively high percentage fit $(70-80 \%)$ in each PC suggests that the model class selected adequately represents this data.

Finally, cytokine weights were analyzed for outliers to uncover which, if any, cytokines have a significantly greater contribution to PC 4. Weights for Interleukin (IL)-4 and IL-10 were greater than one half of the interquartile range, indicating that they may have significantly more influence in PC 4 than the other cytokines.

\section{Discussion}

In this paper, we attempt to capture the interaction dynamics of 18 cytokines in response to a graded maximal exercise challenge and compare these across two groups of male Gulf War era veterans: one group consisting of health sedentary veterans and a second group consisting of veterans from Operation Desert Storm now suffering from Gulf War Illness (GWI), a complex constellation of immune, metabolic and cognitive symptoms thought to present as a result of low-level exposures to battlefield neurotoxins. Here we deconstruct and represent at increasingly finer levels of resolution the interaction dynamics linking 18 cytokines expressed in response to maximal exercise using a linear PCA model. This differs from a more conventional univariate analysis of individual cytokines in that it focuses on coexpression patterns linking multiple cytokines in the context of one another. By virtue of this aggregation across multiple cytokines we benefit from a de-noising effect. We also benefit from the ability to decompose the dynamics of the system into various levels of detail similar in some ways to a form of detrending where one removes low frequency carrier waves to focus on detailed fluctuations that would otherwise be lost.

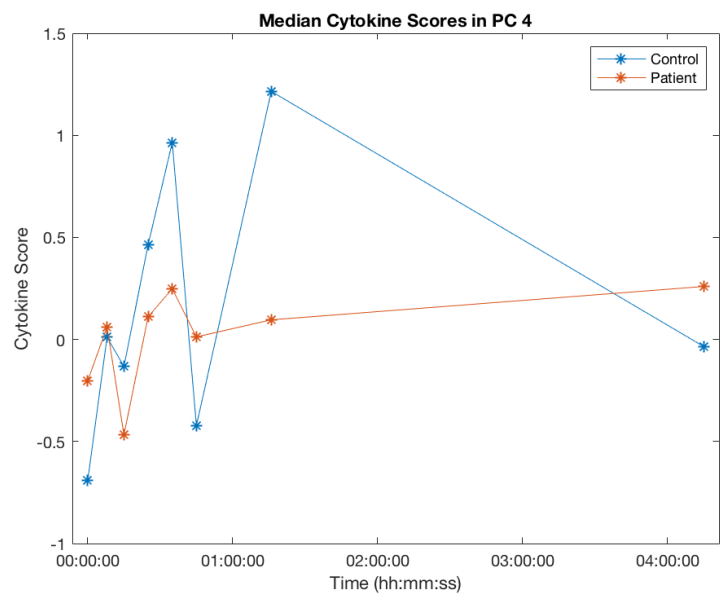

Figure 4: The median raw scores in PC 4 for the control and GWI samples. Median raw scores appear to oscillate less in GWI than in control subjects, in this response is more heavily dampened.

Table 3: Null p-values for the 5 model parameters in each of the PCs. There are two parameters where p-values are $<0.05$ (in bold), namely the dampening coefficient $\zeta$ in PC 4 and the overall gain $K_{p}$ in PC 9. At the resolution of PC 4, the coefficient $\zeta$ is significantly different between groups.

\begin{tabular}{lccccc}
$\mathbf{P C}$ & $\boldsymbol{K}_{\boldsymbol{p}}$ & $\boldsymbol{T}_{\boldsymbol{w}}$ & $\boldsymbol{\zeta}$ & $\boldsymbol{T}_{\boldsymbol{p} \mathbf{3}}$ & $\boldsymbol{T}_{\boldsymbol{z}}$ \\
\hline 1 & 0.78 & 0.73 & 0.56 & 0.32 & 0.78 \\
2 & 0.93 & 0.69 & 0.88 & 0.60 & 0.31 \\
3 & 0.52 & 0.93 & 0.93 & 0.23 & 0.16 \\
4 & 0.21 & 0.31 & $\mathbf{0 . 0 1}$ & 0.98 & 0.46 \\
5 & 0.17 & 0.88 & 0.25 & 0.67 & 0.73 \\
6 & 0.48 & 0.19 & 0.52 & 0.31 & 1.00 \\
7 & 0.83 & 0.98 & 0.60 & 0.69 & 0.24 \\
8 & 0.44 & 0.73 & 0.12 & 0.83 & 0.85 \\
9 & $\mathbf{0 . 0 2}$ & 0.60 & 0.41 & 0.83 & 0.85 \\
10 & 0.98 & 1.00 & 0.69 & 0.69 & 0.73 \\
11 & 0.93 & 0.50 & 0.95 & 0.78 & 0.18 \\
12 & 0.12 & 0.23 & 0.23 & 0.56 & 0.95
\end{tabular}




$\begin{array}{llllll}13 & 0.69 & 0.15 & 0.50 & 0.60 & 0.56 \\ 14 & 0.48 & 0.06 & 0.08 & 0.06 & 0.73 \\ 15 & 0.56 & 0.48 & 0.25 & 0.76 & 0.08 \\ 16 & 0.52 & 0.41 & 0.90 & 0.27 & 0.17 \\ 17 & 0.78 & 0.98 & 1.00 & 0.06 & 0.42 \\ 18 & 0.73 & 0.05 & 0.62 & 0.78 & 0.73\end{array}$

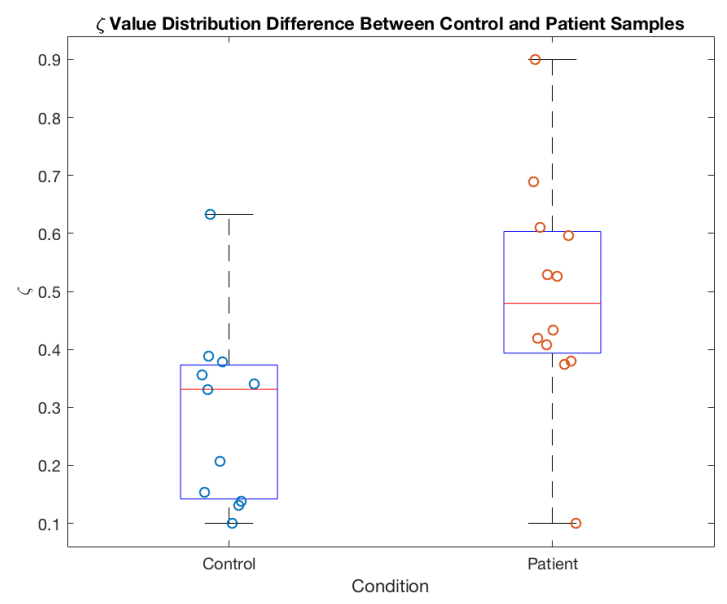

Figure 5: A box plot of the distributions of the $\zeta$ parameters in PC 4 for control and GWI subjects. The $\zeta$ values for GWI are higher than those for the control group indicating a more heavily dampened immune response dynamic in GWI. The null p-value of the Mann-Whitney $U$ test comparing these two distributions is 0.01 , indicating that the oscillatory dynamics are significantly different between groups at this level of resolution.

As the PCA component vectors are extracted from all data regardless of clinical phenotype, these features represent coexpression patterns shared by both subject groups. Differences between groups would then manifest as differences in the overall expression and response dynamics of the feature scores. The response dynamics of each individual subject at these different levels of detail were captured as parameter values in a feedback control model consisting of a third order process model and a conventional PID controller. Median fit to data of 50\% (NRMSE) or better was obtained, with most models explaining $65-85 \%$ of the recorded response. Analysis of model parameters suggested that significant differences in the dampening coefficient existed between groups in a specific co-expression pattern, PC 4, that alone explained over $8 \%$ of the variability in the 18 cytokines. Examination of this co-expression pattern indicated an inordinately high contribution of changes in anti-inflammatory cytokines IL-4 and IL-10, and opposite changes in the inflammatory cytokine Tumor Necrosis Factor (TNF)- $\alpha$. Not a cytokine typically associated with exercise response, IL-4 has nonetheless been shown to mediate the analgesia produced at low-intensity exercise by modulating peripheral and central neuroimmune responses in mice with neuropathic pain [19]. It is interesting to note that no significant differences in immune feedback dynamics were found in the first 3 co-expression features each capturing $19 \%, 14 \%$ and $10 \%$ of cytokine coexpression respectively. In other words, roughly $40 \%$ of cytokine co-expression dynamics in 18 cytokines were essentially conserved across groups underscoring the shared physiology in this group of male subjects in a narrow age group and sharing a common military service history. These similarities notwithstanding, the groups separate quite readily when we examine the oscillatory behavior in the ratio of anti-inflammatory to inflammatory cytokine expression, in particular as they manifest in a cytokine not normally associated with exercise such as IL-4 [12] (see Figure 6).

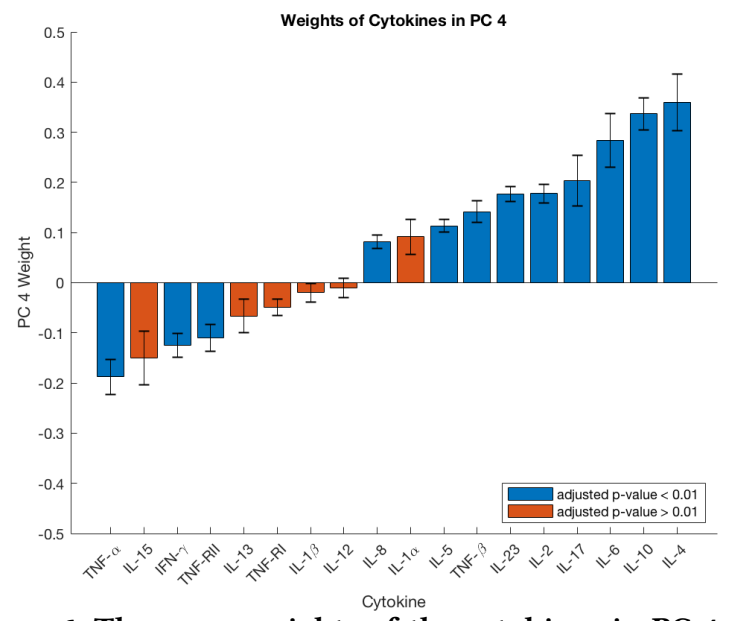

Figure 6: The mean weights of the cytokines in PC 4. The error bars represent one standard error from the mean. Blue (red) bars have a multiple hypothesis corrected p-value $<0.01$ ( $\mathrm{p}$-value $>0.01$ ) from a single sided t-test, indicating that those cytokines (do not) contribute to the PC significantly. Using outlier analysis, IL-4 and IL-10 are considered to have a significantly greater contribution to PC 4 than the other cytokines.

Differences such as these are consistent with a study by Aschbacher et al. (2012) where the parameters of a dynamic model fit to diurnal changes in hormone levels in Chronic Fatigue Syndrome and Fibromyalgia identified subgroups of these diseases [20]. Finally, it is also important to remember that this analysis focused on common cytokine constructs. It is possible that new unique cytokine signaling patterns emerge as a result of this illness that support adaptive responses unique to the GWI group. Evidence of such illness-specific differences in cytokine signaling patterns have been observed and reported previously by our group [21]. Not only does this analysis provide a compelling cytokine signature for GWI, but the methodology presented here may be used to uncover other signatures of complex immune phenotypes.

\section{ACKNOWLEDGMENTS}

This work was supported by Rochester Regional Health in conjunction with the US Department of Defense Congressionally Directed Medical Research Program (CDMRP) http://cdmrp.army.mil/ under awards GW080152 (Klimas - PI), GW093042 (Broderick - PI), GW140142 (Broderick/Craddock - PI; Whitley Partnering PI). Additional funding was provided under 
Merit Awards from the US Department of Veterans Affairs (Klimas, Fletcher - PI). The authors also extend special thanks to Fanny Collado RN, Liz Elizabeth Balbin MS and Jeff Jeffry Cournoyer MS, of the Miami VAMC and Nova Southeastern Institute for Neuroimmune Medicine for their support.

\section{Mandatory Disclaimer:}

The opinions and assertions contained herein are the private views of the authors and are not to be construed as official or as reflecting the views of the Department of Defense.

\section{REFERENCES}

[1.] Fukuda, Keiji, Nisenbaum, Rosane, and Stewart, Geraldine. Chronic Multisymptom Illness Affecting Air Force Veterans of the Gulf War. JAMA, 280 (Sep. 1998), 981.

[ 2.] Medicine, Institute. Gulf War and Health: Volume 8: Update of Health Effects of Serving in the Gulf War. The National Academies Press, Washington, 2010.

[ 3.] Li, B., Mahan, C. M., Kang, H. K., Eisen, S. A., and Engel, C. C. Longitudinal Health Study of US 1991 Gulf War Veterans: Changes in Health Status at 10 -Year Follow-up. American Journal of Epidemiology, 174 (July 2011), 761-768.

[ 4.] Chao, Linda L., Rothlind, Johannes C., Cardenas, Valerie A., Meyerhoff, Dieter J., and Weiner, Michael W. Effects of lowlevel exposure to sarin and cyclosarin during the 1991 Gulf War on brain function and brain structure in US veterans. NeuroToxicology, 31 (Sep. 2010), 493-501.

[ 5.] Golomb, B. A. Acetylcholinesterase inhibitors and Gulf War illnesses. Proceedings of the National Academy of Sciences, 105 (Mar. 2008), 4295-4300.

[ 6.] Whistler, Toni, Fletcher, Mary Ann, Lonergan, William et al. Impaired immune function in Gulf War Illness. BMC Medical Genomics, 2 (Mar. 2009).

[ 7.] Medicine, Institute. Military Strategies for Sustainment of Nutrition and Immune Function in the Field. National Academies Press, 1999.

[ 8.] Dembic, Zlatko. Chapter 1 - Introduction-Common Features About Cytokines. In Dembic, Zlatko, ed., The Cytokines of the Immune System. Academic Press, Amsterdam, 2015.

[ 9.] Pedersen, Bente Klarlund, Ostrowski, Kenneth, Rohde, Thomas, and Bruunsgaard, Helle. The cytokine response to strenuous exercise. Canadian fournal of Physiology and Pharmacology, 76 (May 1998), 505-511.

[10.] Suzuki, Katsuhiko. Cytokine Response to Exercise and Its Modulation. Antioxidants, 7 (Jan. 2018), 17.

[11.] Zhou, Xin, Fragala, Maren S., McElhaney, Janet E., and Kuchel, George A. Conceptual and methodological issues relevant to cytokine and inflammatory marker measurements in clinical research. Current Opinion in Clinical Nutrition and Metabolic Care, 13 (Sep. 2010), 541-547.
[12.] Pedersen, Bente Klarlund and Toft, Anders Dyhr. Effects of exercise on lymphocytes and cytokines. British fournal of Sports Medicine, 34 (2000), 246-251.

[13.] Pearson, Karl. LIII. On lines and planes of closest fit to systems of points in space. The London, Edinburgh, and Dublin Philosophical Magazine and Journal of Science, 2 (Nov. 1901), 559-572.

[ 14.] Fritsch, F. N. and Butland, J. A Method for Constructing Local Monotone Piecewise Cubic Interpolants. SIAM fournal on Scientific and Statistical Computing, 5 (June 1984), 300-304.

[ 15.] Kuby, Janis. Immunology. W.H. Freeman and Company, New York, 1994

[ 16.] Hart, Y., Antebi, Y. E., Mayo, A. E., Friedman, N., and Alon, U. Design principles of cell circuits with paradoxical components. Proceedings of the National Academy of Sciences, 109 (May 2012), 8346-8351.

[17.] Mann, H. B. and Whitney, D. R. On a Test of Whether one of Two Random Variables is Stochastically Larger than the Other. The Annals of Mathematical Statistics, 18 (Mar. 1947), 50-60.

[ 18.] Benjamini, Yoav and Hochberg, Yosef. Controlling the false discovery rate: a practical and powerful approach to multiple testing. Fournal of the Royal statistical society: series $B$ (Methodological), 57 (1995), 289-300.

[ 19.] Bobinski, Franciane, Teixeira, Juliana Maia, Sluka, Kathleen Anne, and Santos, Adair Roberto Soares. Interleukin-4 mediates the analgesia produced by low-intensity exercise in mice with neuropathic pain. PAIN (Nov. 2017), 1.

[ 20.] Aschbacher, Kirstin, Adam, Emma K., Crofford, Leslie J., Kemeny, Margaret E., Demitrack, Mark A., and Ben-Zvi, Amos. Linking disease symptoms and subtypes with personalized systems-based phenotypes: A proof of concept study. Brain, Behavior, and Immunity, 26 (October 2012), 1047-1056.

[21.] Broderick, Gordon, Kreitz, Andrea, Fuite, Jim, Fletcher, Mary Ann, Vernon, Suzanne D., and Klimas, Nancy. A pilot study of immune network remodeling under challenge in Gulf War Illness. Brain, Behavior, and Immunity, 25 (Feb. 2011), 302-313. 